\title{
Study on Network Characteristics of Surface-to-air-missile Defence System
}

\author{
ZHAO Yan, WANG Ning, HE Guangjun \\ Air Force Engineering University, Air and Missile Defense College, Xi`an 710051 China
}

Keywords: Surface-to-air-missile; defence system; missile netting; small world characteristic

\begin{abstract}
Aiming at the poor reliability of targets destroy for single base surface-to-air-missile weapon system, and the existing shortcomings of anti-destroying ability for missile networking system, this paper research on system level surface-to-air-missile defense system network features. On the analysis of the missile launching area boundary, the kill probability, the operational time and threat level, the system level surface-to-air-missile defense system network model is established. Combined with the average path length and the local clustering coefficient complex network science, the network structure is optimized. It is verified by simulation experiments that the optimized defense system network model with smaller average path length and large local clustering coefficient, just as a small world network features. This structure improves the reliability and anti-destroying ability of surface-to-air-missile defense system.
\end{abstract}

\section{Introduction}

With the increasing of the degree of informatization and networking, the modern war form have fundamental changed. Especially depended on the integration command and control system, the military powers carry on the standoff precision strike, with the electronic countermeasure technology, stealth technology, to improving the survival ability of weapon system. It bring serious challenges for traditional surface-to-air defense weapon system ${ }^{[1-3]}$.

The center network framework was established in [4]. The information share and communication between the weapon systems is achieved, and the regional intercept ability of ballistic missile defense system was improved. The reliability of air defense weapon system network was studied in [5], from the three layers, the networking principle of weapon system is analyzed, and the reliability of the system is proved under different network structure. It is demonstrated that the anti-jamming and anti-stealth of the system is obviously improved by the simulation. After analyzing the literature, the networking air defense weapon system can greatly improve the precision and reliability. But the resist destructive capacity of complicated system network is poor. When the center node of network is destroyed, the entire network will be paralyzed.

Therefore, based on the existing research, this paper proposes the multilayer regional surface-to-air-missile defense system network model, and verified that the network model with small world effect, that is network with small average distance and large clustering coefficient. The performance of the system network is improved such as system reliability, anti-destroying ability, and so on.

\section{Main factors influencing missile position deployment}

Air defense weapon system position configuration is an important work for air defense combat command. It is great important to defend the point safety. The major factors affecting missile position deployment are kill zone, the launch region and reaction time, and so on.

\subsection{Kill zone}

The kill zone is the battle space, the area of surface-to-air-missile weapon system with no less than specified probability to kill target under specified conditions, including the far, near, high and low field, such as shown in figure 1. 
Fig.1 The canonical schema of the kill zone

In figure 1, the horizontal abfe, the horizontal $d c n m$, the surface abcd, the sector eflk, the spherical lkmn, the vertical surface admke and bcnlf are the high field, the low field, the far field, far and near field, low near field, boundaries of kill zone, respectively.

\subsection{Launch region}

The launch region is the space that makes the target encounter with missile in the kill zone. The far field of launch region $d_{f}$ is missile destroy targets in far field of launch region; the near field of launch region $d_{n}$ is missile destroy targets in near field of launch region.

\subsection{Reaction time and transfer fire shooting time}

The reaction time $t_{r}$ is time interval that is from find target moment to launch the first missile moment of surface-to-air-missile weapon system. The transfer fire shooting time $t_{t}$ is time interval that is from previous missile finished to completion prepares of next missile launch. The time interval is expressed as

$$
t_{t}=t_{r}+t_{p}
$$

In Eq.(1), $t_{r}$ is the reaction time, $t_{t}$ is the transfer fire shooting time.

\section{Networking model of surface-to-air missile defense system}

The networking combat of surface-to-air missile defense system is composed of multiple (kinds of) detection and guidance equipment and interceptors. The command and control nodes are connected by distributed network links. The combat effectiveness of the different platforms is maximized in different combat environment.

The network model of surface-to-air-missile defense system has three layers networking model. The first layer is decision layer, the 2nd layer is command and control layer, and third layer is combat, as shown in figure 2.

In figure 2, the decision layer has $P_{i}(i=1,2, \cdots, n)$ nodes, the command and control layer has $C_{i j}$ $(i=1,2, \cdots, n, \quad j=1,2, \cdots, m)$ nodes, the combat has $W_{i j k}(i=1,2, \cdots, n, j=1,2, \cdots, m, \quad k=1,2, \cdots, l)$ nodes. One node connects with other adjacent four nodes in the same layer.

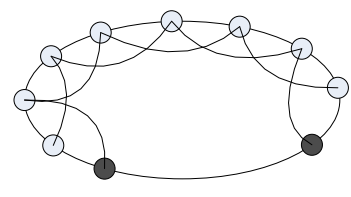

Fig.2 The model of defence network on system level 


\section{Parameters of the defense system networking model}

\subsection{The degree distribution of the model}

The node degree is the number that the node connected to the other nodes ${ }^{[6-8]}$. The node degree distribution is the node degrees obey the distribution, usually expressed by function $P(k)$. Poisson distribution is adopted in this paper.

$$
P(x=k)=\frac{\lambda^{k}}{k !} e^{-\lambda}
$$

\subsection{Average path length of the model}

In the network with $n$ nodes, the average path length is ${ }^{[6-8]}$

$$
l=\frac{1}{\frac{1}{2} n(n+1)} \sum_{i \geq j} d_{i j}
$$

In Eq.(3), $d_{i j}$ is the shortest distance from node $i$ to $j$. If the shortest distance between two nodes is infinite, then the Eq.(4) can calculate the average path length to eliminate the influence of infinity.

$$
l^{-1}=\frac{1}{\frac{1}{2} n(n+1)} \sum_{i \geq j} d_{i j}^{-1}
$$

\subsection{Clustering coefficient of the model}

The clustering coefficient is used to measure group of the network ${ }^{[6-8]}$.

$$
c=\frac{1}{n} \sum_{i} c_{i}
$$

there, $c_{i}$ is the local clustering coefficient of the node $i$.

$$
c_{i}=\frac{2 E_{i}}{k_{i}\left(k_{i}-1\right)}
$$

In Eq.(6), $k_{i}$ is the edge of connection with node $i, E_{i}$ is the number of the actual connection edge.

\section{Simulation experiments and analysis}

\subsection{Simulation parameters}

Set the decision layer has three sites in the network, each node connect with 3 nodes in C2 layer, and each node in C2 layer charges with three nodes in combat. Then there are 39 nodes total in the system, as shown in figure 3 . In order to optimized the network, the connection probability is set to $p_{i}=0.2$. The optimized network structure is shown as in figure 4 .

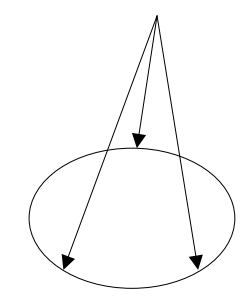

Fig.3 The tree-like structures before network optimized 


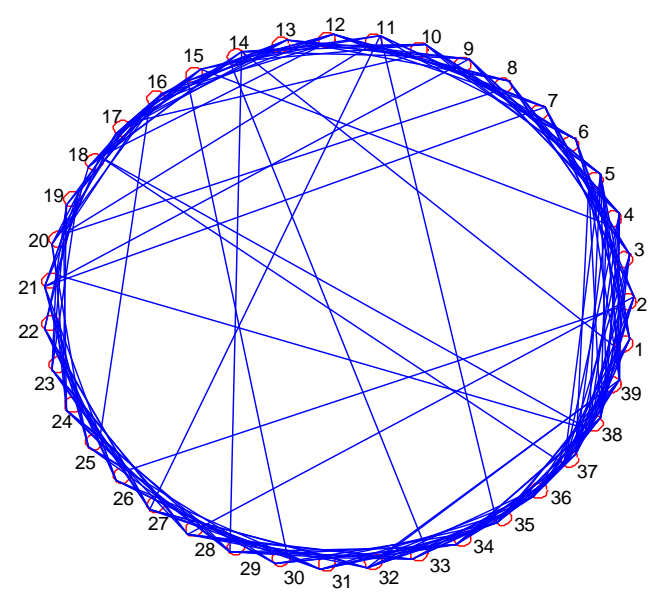

Fig.4 The structures after network optimized

\subsection{Simulation analysis}

The shortest path $d_{i j}$ can be calculate by Floyd algorithm, then the average path length is get by Eq.(4). the average path length is compared between original net $(l(p))$ and optimized net $(l(0))$. The circle is $l(p) / l(0)$ in figure 5 . The curve is the ratio of curve fitting. We get

(i) The $l(p) / l(0)$ is less than $1, l(0)$ is bigger than $l(p)$, the average path length of optimized net is shorter than original net's.

(ii) The ratio curve function is concave function, the bigger ratio has lower probability, the small ratio has higher probability. It is more that $l(p)$ is far less than $l(0)$. the optimized system network has short average path length than original net.

The clustering coefficient is calculate by Eq.(6). The clustering coefficient is compared between original net $(c(p))$ and optimized net $(c(0))$. The circle is $c(p) / c(0)$ in figure 6 .

(i) The $c(p) / c(0)$ is less than $1, c(0)$ is bigger than $c(p)$, the clustering coefficient of optimized net is shorter than original net's.

(ii) The ratio curve function is convex function, but the bigger ratio has higher probability, the small ratio has lower probability. It is less that $c(p)$ is far less than $c(0)$. The optimized system network has relatively short clustering coefficient than original net.

From the above analysis, the original network has larger the average path length and clustering coefficient, the optimized network has shorter the average path length and relatively larger clustering coefficient with characteristics of small-world networks.

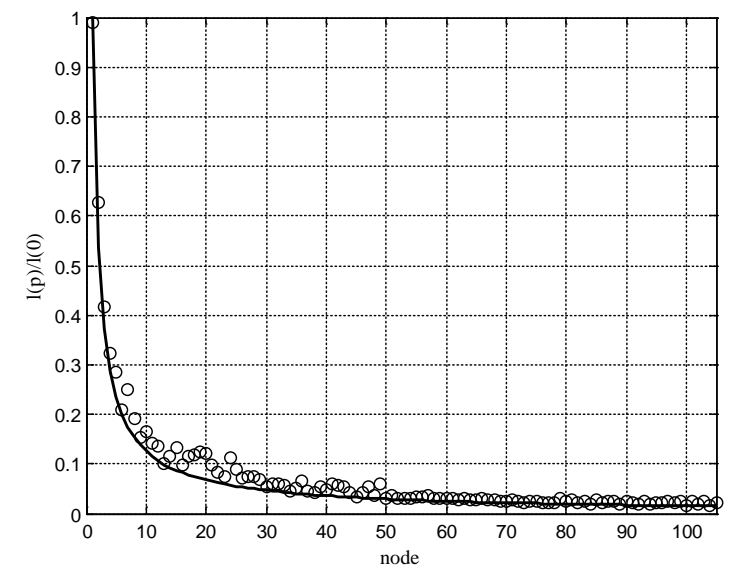

Fig. 5 The compared curve of average path length 


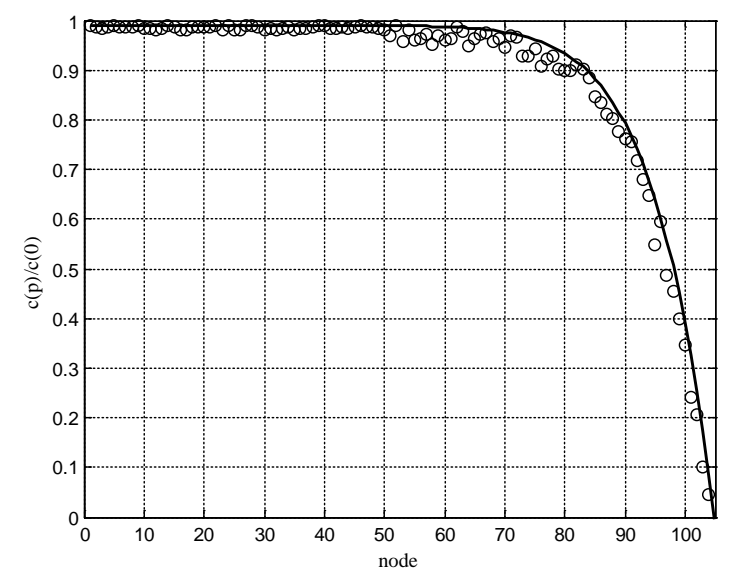

Fig.6 The compared curve of clustering coefficient

\section{Conclusions}

In this paper, the system level surface-to-air-missile defense system network features is researched. Combined with the average path length and the local clustering coefficient complex network science, the network structure is optimized. The simulation experiment is shown that the optimized defense system network has a small world network features. This structure improves the reliability and anti-destroying ability of surface-to-air-missile defense system.

\section{Reference:}

[1] WANG Xi, LI Jianxun.The Analysis on the Networked Operational Patterns of Surface-Based Air Defense Missile System[J]. Aerospace Control, 2012, 30(5): 25-29. (in Chinese)

[2] MA Cenrui, LONG Guangzheng, YANG Yun. Evaluating Model on Effectiveness of Network Defense Missile Based on LMBP Neural Network[J]. Lecture Notes in Computer Science, 2011, 6675(1): 557-564.

[3] XU Pingao. Requirement Analysis of Modern Short Range Air Defence Missiles for End Defence[J]. Modern Defence Technology, 2003, 31(4): 1-7. (in Chinese)

[4] Michael N. Gagnon, John Truelove, Apu Kapadia, etc. Towards Net-Centric Cyber Survivability for Ballistic Missile Defense[J]. Lecture Notes in Computer Science, 2010, 6150(1):125-141.

[5] LONG Guangzheng, LIU Ming, LI Lingxiang, etc. A Study on Reliability Modeling of Air-defense Missile Weapon Systems Networking[J]. Journal of Air Force Engineering University(Natural Science Edition), 2006, 7(5):16-18. (in Chinese)

[6] A. Derzsia, N. Derzsya, E. Káptalana, etc. Topology of the Erasmus student mobility network[J]. Physica A, 2011, 390: 2601-2610.

[7] LI Bing, CHEN Guanrong, CHOW Tommy W.S. Naming Game with Multiple Hearers[J]. Commun Nonlinear Sci Numer Simulat, 2013, 18: 1214-1228.

[8] Subhasis Das, Sachin Patkar. A Compact Gaussian Random Number Generator for Small Word Lengths[J]. Lecture Notes in Computer Science, 2011, 6578(1):88-93. 